\title{
Recruitment of Underrepresented Minority Researchers into HIV Prevention Research: The HIV Prevention Trials Network Scholars Program
}

\author{
Sten H. Vermund, Erica L. Hamilton, Sam B. Griffith,' Larissa Jennings, \\ Typhanye V. Dyer, Kenneth Mayer, and Darrell Wheeler ${ }^{6}$
}

\begin{abstract}
Most U.S. investigators in the HIV Prevention Trials Network (HPTN) have been of majority race/ethnicity and sexual orientation. Research participants, in contrast, have been disproportionately from racial/ethnic minorities and men who have sex with men (MSM), reflecting the U.S. epidemic. We initiated and subsequently evaluated the HPTN Scholars Program that mentors early career investigators from underrepresented minority groups. Scholars were affiliated with the HPTN for 12-18 months, mentored by a senior researcher to analyze HPTN study data. Participation in scientific committees, trainings, protocol teams, and advisory groups was facilitated, followed by evaluative exit surveys. Twenty-six trainees have produced 17 peer-reviewed articles to date. Research topics typically explored health disparities and HIV prevention among black and Hispanic MSM and at-risk black women. Most scholars ( $81 \%$ in the first five cohorts) continued HIV research after program completion. Alumni reported program-related career benefits and subsequent funding successes. Their feedback also suggested that we must improve the scholars' abilities to engage new research protocols that are developed within the network. Mentored engagement can nurture the professional development of young researchers from racial/ethnic and sexual minority communities. Minority scientists can benefit from training and mentoring within research consortia, whereas the network research benefits from perspectives of underrepresented minority scientists.
\end{abstract}

Keywords: training, mentorship, professional development, minority researchers, lesbian-gay-bisexualtransgender, health research workforce

\section{Introduction}

T HE HIV EPIDEMIC swept across the United States in the 1980s and a cadre of motivated researchers tackled its urgent biological, behavioral, and social issues. The first recognized cases were in white men who had sex with men (MSM). In subsequent years, the racial/ethnic mix shifted to affect disproportionately persons from minority populations, with black and Hispanic (i.e., Latino/Latina) MSM and women at highest risk of infection. Nearly two-thirds $(65 \%)$ of newly diagnosed cases in the United States in 2010 were among blacks or Hispanics, and $63 \%$ were among MSM. ${ }^{1}$

Investigators who are representative of HIV at-risk populations can be expected to have many advantages in developing and conducting HIV prevention research. For example, minority investigators may have insights from their own experiences, may better understand concerns of historically oppressed communities and individuals, and may better anticipate key issues of racial and cultural inclusion and community sensitivities about research. Social justice issues that frame the

\footnotetext{
${ }^{1}$ Department of Epidemiology of Microbial Diseases, Center for Interdisciplinary Research on AIDS, Yale School of Public Health, New Haven, Connecticut.

${ }^{2}$ FHI 360, Durham, North Carolina.

${ }^{3}$ Social and Behavioral Interventions Program, Department of International Health, Bloomberg School of Public Health, Johns Hopkins University, Baltimore, Maryland.

${ }^{4}$ Department of Epidemiology and Biostatistics, University of Maryland, College Park, Maryland.

${ }^{5}$ Fenway Health, Beth Israel Deaconess Medical Center, Harvard Medical School, Boston, Massachusetts.

${ }^{6}$ School of Social Welfare, University at Albany State University of New York, Albany, New York.

(c) Sten H. Vermund et al. 2018; Published by Mary Ann Liebert, Inc. This article is available under the Creative Commons License CC-BYNC (http://creativecommons.org/licenses/by-nc/4.0). This license permits non-commercial use, distribution and reproduction in any medium, provided the original work is properly cited. Permission only needs to be obtained for commercial use and can be done via RightsLink.
} 
context for HIV among racial/ethnic minorities include educational and economic disparities, housing conditions, discrimination, racism, homophobia, access to healthcare, and historic exclusions of minority investigators from mainstream academic or public health research opportunities. Increasing the number of historically underrepresented or disenfranchised investigators in HIV research can be expected to increase attention to important contextual issues.

The HIV Prevention Trials Network (HPTN) developed a special initiative, the HPTN Scholars Program, to recruit, engage, train, and mentor young minority investigators within a large HIV prevention clinical trials collaborative group. The goal of this article is to describe the HPTN Scholars Program and its influence on the professional lives of its alumni and the network.

\section{Materials and Methods}

\section{Background to the HPTN}

The HPTN conducts randomized controlled trials (RCTs) to assess the effectiveness of HIV prevention strategies and technologies in reducing adult and adolescent HIV transmission., ${ }^{2,3}$ Smaller vanguard studies may precede an RCT to address questions needed for trial design and to prepare study sites. Key topics currently being studied within the HPTN include the use of antiretroviral drugs to reduce HIV transmission between adults in two ways: (1) by reducing infectiousness among HIVinfected persons taking combination antiretroviral therapy, termed "treatment as prevention," and (2) by reducing susceptibility among HIV-uninfected persons through preexposure prophylaxis (PrEP) with oral or depot antiretroviral drugs. $^{4-8}$ Another top priority of HPTN work is the use of integrated strategies that combine effective biomedical, behavioral, and structural elements to reduce transmission. ${ }^{9-12}$

\section{The HPTN Scholars Program}

Our HPTN Scholars Program followed a successful mentored medical science opportunity for underrepresented racial/ethnic minority students offered by the HIV Vaccine Trials Network (HVTN) through their Research and Mentorship Program (RAMP). RAMP supports medical students to conduct either summer (2-4 months) or 9-12-month-long projects within the HVTN. ${ }^{13}$ The HPTN Scholars Program differs from RAMP by supporting both physicians and postdoctoral level researchers early in their careers (rather than medical students), and by explicitly encouraging applicants from sexual minority groups as well as racial/ethnic minority groups to apply. A similar program to nurture junior investigators in vaccine laboratory science has subsequently been established by the HVTN and the Center for HIV/AIDS Vaccine Immunology. ${ }^{14}$

Individuals eligible for the HPTN Scholars Program are earlystage investigators (e.g., doctoral degree completed within 10 years of application) who self-identify as an underrepresented racial/ethnic minority (e.g., black or African American, Hispanic, Latino/Latina, American Indian, Alaskan Native, Native Hawaiian, or other Pacific Islander) in the health-related sciences. Persons of minority sexual orientations are actively encouraged to apply. Applicants cannot have received an investigatorinitiated R01 or similar NIH grant. To support participation in the Scholars Program, the scholar's academic institution is com- pensated for $10 \%-30 \%$ of the scholar's annual salary, and receives funding for research supplies and travel to two HPTN annual meetings, one mid-program scholars workshop, and one other HIV-related conference. This budget, of up to U.S. $\$ 95,000$ (total), is submitted by the scholar during the application phase.

Scholar applicants are accepted into a cohort for a period of 18 months. From program inception (2010) and through Cohort 3, scholars were chosen for a 1-year term and then offered the opportunity to apply for a second year. The switch to an 18-month period was made during Cohort 4 (2013) to provide scholars more time to complete their research, move their articles through the HPTN review process, and submit for publication. The newest cohort as of this writing, the 2017-2018 scholars, represents Cohort 8.

The HPTN Scholars Program's requirements are that scholars (1) develop a research project that uses previously unanalyzed data from HPTN studies, (2) complete the approved project within the program period, (3) present their findings at the HPTN annual meeting, and (4) submit an article for peer-reviewed publication, typically as the lead author. These requirements relate to program goals, for scholars are to (1) acquire new knowledge, skills, and connections to further their careers as independent investigators in HIV science, (2) become knowledgeable of the process of conducting rigorous research in NIH-funded HIV networks, and (3) have the opportunity to build research linkages within the prevention research context of the HPTN.

HPTN scholar mentoring. The keystone element of the HPTN Scholars Program is mentoring, defined for this program as: a relationship that gives more experienced researchers (the mentor) the opportunity to provide guidance and share their professional skills and experience with less experienced researchers (the scholar) with the goal of helping scholars complete their scholarship project and develop their knowledge, skills, and connections. Matching of scholars with mentors has been based on a matrix of possibilities. Some mentors suggest to their own junior colleagues that the HPTN Scholars Program might be of interest. Some prospective scholars started the matching process by indicating specific interests to program leaders (S.H.V. and D.W.) or to other HPTN investigators who, in turn, suggested appropriate mentors. Some scholars have contacted the Program Manager (E.L.H.), who has linked them with an HPTN investigator, or have simply reached out to HPTN investigators whose research was of interest to them.

The specifics of any mentoring relationship depend upon many factors, including where the scholar is in his or her career; what knowledge, skills, and connections the scholar seeks and the mentor has to share; the personalities and availability of the scholar and mentor; and the motivation of the scholar and mentor. A critical implicit goal in the mentored relationship is to assist the scholar in navigating the complex systems of network research and to promote self-efficacy that will enable scholars to move toward longer term success and retention in HIV prevention research. Mentors graciously volunteer their time and expertise to foster these future HIV prevention scientists.

Mentors and scholars identify the highest priority areas in which the scholars' individual knowledge, skills, and connections can be improved at the beginning of the scholars program. They interact on a regular basis throughout the duration of the scholarship, scaling up to more frequent and intensely focused meetings when certain milestones in the 
project approach (e.g., a scholar's presentation at the HPTN annual meeting). Mentors and scholars also participate in periodic structured discussions to set goals and ensure substantial progress. The ideal mentor provides appropriate guidance, exposure, introductions, and support to help broaden a scholar's range of experience and knowledge as an HIV researcher. Mentors help broker opportunities for continued scholar engagement within the HPTN through linkage to community and HPTN scientific committees, such as the MSM, substance use, and women-at-risk scientific committees and the HPTN Black Caucus, a community/investigator consortium that focuses on racial/ethnic and sexual orientation issues. All of the mentorship activities are focused on the overall goal of preparing the scholar for a long-term successful HIV research career.

Scholar recruitment, orientation, and training. Recruitment, application review, and acceptance into the scholars program takes place annually. The program is advertised through public health and medical schools and through HPTN-related communications. Alumni also participate in outreach and promotion of the scholars program and contribute to the visibility of the scholars program on social media.

After selections are made, orientation begins each year at the HPTN annual meeting, and scholars are introduced to one another and to HPTN leaders. For some, this is also the first opportunity they have to meet face-to-face with the mentors who have agreed to work with them. By attending the plenary sessions, protocol team meetings, and working group sessions of the annual meeting, scholars obtain comprehensive exposure to the work, culture, and personnel of the network. During scholar-specific training, scholars learn about the history, structure, and science generation process of the network, as well as review the expectations of scholars during their tenure. Program leadership reviews a timeline of deliverables and discusses expectations for mentorship, participation in calls and meetings, submission of contracts and invoices, and dissemination of findings from the scholars' secondary analyses. A program manual, a mentorship manual, and a program timeline are provided to guide this review of expectations and as reference materials during the rest of the program period.

Also at the annual meeting, new scholars meet scholars who have already been in the program for a year, as well as alumni who are continuing their work within the network. These interactions allow new scholars to receive practical advice on how best to take advantage of the program from "near-peers," a secondary mentorship process that has often continued well beyond the annual meeting. Scholars present their proposed analyses during the meeting to a group that includes other new and continuing scholars, mentors, and program leaders. Feedback during this session is used by scholars to strengthen their proposals.

Once the scholars begin work on their secondary analysis project, much of their professional growth comes through the process of working with their mentors. The mentor helps refine ideas and plan analyses. She or he also provides regular feedback during research conduct and writing. The mentor often will facilitate connections between the scholar and NIH staff or other researchers working on the protocol or in the mentor's professional network. Mentors help scholars think through next steps in their career path and what they need to be doing to ensure their advancement. Scholars' research is nurtured, too, by the statisticians from the HPTN's Statistical and Data Management Center (SDMC) who review the scholar's data analysis plan to ensure feasibility and statistical accuracy.

Approximately 6 months after beginning the program, scholars convene in Seattle, Washington, at the SDMC, housed at the Statistical Center for HIV/AIDS Research and Prevention within the Fred Hutchinson Cancer Research Center, for a 2-day professional development workshop (scholars workshop). The training workshop focuses on statistical analysis - including how to write analysis plans - as well as key topics in clinical trial design, conduct and analysis, how to address missing data in research studies, and other topics, provided by senior HPTN statisticians. Additional training is also provided on topics particularly helpful to early career minority researchers, such as article writing and how to pursue and maintain an HIV research career.

Evaluation. A program exit evaluation is completed by each scholar to inform subsequent iterations of the program. To further examine the influence of the scholars program on the careers of scholars who have completed the program, we asked alumni from Cohorts 1, 2, and 3 what they saw as the benefits of the program, looking back 2-4 years after completion of the program. Twelve of 13 scholars who stay in touch with the HPTN were approached, 11 of whom responded (92\%). The email solicitations stated that the scholars program managers were evaluating the program and sought to document scholar achievements. The evaluation was exempt from Institutional Review Board review.

The evaluation focused on three programmatic outcomes. First are products from the scholars' projects (e.g., peerreviewed publications, presentations, and posters), notably their published articles. Second, are the other opportunities the scholars have had that they attributed to the scholars program (e.g., invitations to meetings or other events, securing funding based on their HPTN work). Third represents benefits toward professional advancement, notably the evolution in professional development plans based on experiences with the scholars program (e.g., skills acquisition, promotion, and networking). Finally, we assessed whether the scholars continued in an HIV/AIDS research field after completion of the program.

\section{Results}

\section{Scholars program feedback}

There have now been 26 scholars who have completed their HPTN training (Table 1), with 12 more in the 20162017 scholars group (Cohort 7) and the 2017-2018 scholars group (Cohort 8). Two scholars, both from Cohort 1, have been continuously engaged in the HPTN for $>5$ years, including attending and presenting at HPTN annual meetings, presenting in webinars, writing NIH grants using HPTN data, and serving on protocol teams for new HPTN studies.

Given the lack of sufficient follow-up time for later cohorts, we only surveyed Cohorts $1-3$. Program feedback has been overwhelmingly positive. Scholars' feedback strongly suggested that having the HPTN scholars award was helpful when applying for faculty positions by demonstrating prior success in attaining funding. Participation in the scholars 
Table 1. Background of Scholars in the Mentored HIV Prevention Trials Network Scholars

Program at Time of Application (2010-2015), COHORTS $1-6(N=26)$

n $(\%)$

\begin{tabular}{lr}
\hline Self-identified sex & \\
Men & $6(23)$ \\
Women & $20(77)$ \\
Age in years & \\
24-29 & $1(4)$ \\
30-34 & $17(65)$ \\
35-39 & $4(15)$ \\
40-44 & $3(12)$ \\
45-49 & $1(4)$ \\
Self-identified race/ethnicity & \\
Black/African American & $22(85)$ \\
Hispanic/Latino/Latina & $4(15)$ \\
State/Venue of residence & \\
U.S. northeast (Maryland; Washington, DC; & $11(42)$ \\
Massachusetts; New York; Pennsylvania) & \\
U.S. south (Arkansas; Georgia; Mississippi; & $9(35)$ \\
$\quad$ North Carolina; Texas; Virginia) & $3(12)$ \\
U.S. west (California) & $2(8)$ \\
U.S. midwest (Illinois) & $1(4)$ \\
Canada (U.S. citizen) & \\
Scholar project emphasis by study & $4(15)$ \\
Persons who inject drugs (HPTN 037) & $2(8)$ \\
Voluntary testing and counseling (HPTN 043) & $3(12)$ \\
Prevention with positives (HPTN 063) & $8(31)$ \\
Men who have sex with men (HPTN 061) & $8(31)$ \\
Women at risk (HPTN 064) & $1(4)$ \\
Adolescents/youth (HPTN 068) &
\end{tabular}

All HPTN protocols are available at: www.hptn.org/research/ studies, accessed December 9, 2017. We did not collect data on the sexual preferences of HPTN scholars and hence we do not know the number who were sexual minorities, for example, same sex preference or transgender.

HPTN, HIV Prevention Trials Network.

program was perceived to have aided scholars in nearing or obtaining internal departmental approval for promotion. As one 37-year-old African American woman stated, "Having the HPTN Scholars grant was a bonus when I was applying for faculty positions. It demonstrated that I had early success seeking grant funding, and I think enhanced my portfolio as a faculty candidate. I have now been in my current position as an Assistant Professor...for two and half years and am progressing well." This echoed the comments of other program alumni.

Scholars cited mentorship as a highly valued contribution to furthering career and professional development. Other positive outcomes of the program involved inclusion of scholars by mentors in other, non-HPTN-related projects. Mentors were credited for having been critical in fostering the inclusive and supportive environment of the scholars program. Alumni valued the HPTN-funded opportunity to present scholars' work at related conferences, increasing the visibility and support for scholars' research projects. Scholars mentioned that their professional development and writing skills were furthered by the process of working through the analyses and having iterative reviews by study statisticians, publications committees, scholars leaders, and the HPTN Manuscript Review Committee.
Of the 26 HPTN scholars, $9(35 \%)$ were colocated (same city or within $1 \mathrm{~h}$ drive) with their mentors. For persons not colocated, travel was funded for periodic meetings between scholar or mentor to travel to permit face-to-face work. When mentors were not colocated, special efforts were made to encourage conscientious communications and oversight. Since the "distance mentors" were selected specifically for their expertise, scholars only rarely cited the distance from their mentors as a disadvantage within the program. Thanks to a special training program offered at the University of California, San Francisco, several mentors, both colocated and distance, were able to participate in a short course designed to help faculty build their own mentoring skills. We are now helping interested scholar alumni to themselves serve as scientific peer mentors, engaging the National Research Mentoring Network (http://nrmnet.net, accessed December 9, 2017).

Scholar alumni in Cohorts 1-3 who received the survey credited participation in the program as contributing to other benefits, including (1) participation as peer mentors themselves; (2) acceptance into competitive academic programs at their institutions; (3) new contacts with NIH institutes, especially the National Institute of Allergy and Infectious Diseases (NIAID), the National Institute for Mental Health (NIMH), and the National Institute on Drug Abuse (NIDA); and (4) opportunities to collaborate or serve as project directors or principal investigators on HIV prevention grants, including investigator-initiated grants. These scholars noted that they have been invited to contribute to articles with other HPTN investigators as subject area experts in the area of their scholar project. Community involvement, such as participation in the HPTN Black Caucus, was identified as a highlight of scholars' experience, resulting in long-term connections first fostered in the HPTN.

Scholars have also reported participating in additional research development colloquia for junior faculty such as the Robert Wood Johnson Foundation New Connections Program and the NIH Office of Behavioral and Social Sciences Research Summer Institute on Randomized Behavioral Clinical Trials. They have been accepted as postdoctoral fellows at the U.S. Center for Disease Control and Prevention and have assumed positions within diversity-oriented leadership committees and evaluation teams. Four of Cohorts 1-3 former scholars have been awarded NIH K-series career development awards. One former scholar received an NIH small grant (R03) as principal investigator, using HPTN data.

Critical comments received from scholars and mentors have helped in annual reviews and modifications made to the program. Feedback included the scholars' challenges with time management; the part-time nature of the experience did not allow optimal time for the HPTN project. In many cases, scholars reported their inability to perform more complex statistical analyses independently as a limitation of the experience. One scholar from the first cohort did not finish the project and reported feeling unsupported by the program. Another scholar did not feel that data were provided to conduct analyses in a timely manner. Other scholars have bemoaned the short tenure of the program, in that it was initially only 12 months (expanded to 18 months beginning in Cohort 4). Other scholars stated that it has been difficult to maintain a sustained relationship with the HPTN, perceiving a closed-network culture and/or not feeling welcome onto 
protocol teams. Several scholars reported feeling isolated in their home institutions and/or lacking consistent senior mentoring to assist them as they navigated research and/or clinical practice with underserved and other minority groups.

\section{Research outcomes}

As of February 2017, 26 scholars had produced 17 primary-authored, peer-reviewed publications, with 2 in press, ${ }^{12,15-30}$ based upon their scholarship projects, with some having led or contributed to more than one HPTNrelated article. (Cohort 6 , just graduated, has not yet had published work, although several are in review.) The articles have included qualitative, quantitative, and mixed methods research approaches. Scholars have presented project-related posters and oral presentations at scientific meetings of the International AIDS Society, the American Public Health Association, the Conference on Retroviruses and Opportunistic Infections, and the National African American MSM Leadership Conference on HIV/AIDS and other Health Disparities, among others.

\section{Discussion}

It is axiomatic that researchers will strive to train their successors. Mentoring junior scientists is a crucial strategy in that many early cadre HIV prevention researchers will retire in the coming years. Particularly in the field of HIV prevention research, there is a compelling imperative to nurture minority investigators who better reflect the sociodemographic and sexual preference diversity of HIV-infected persons. The HPTN Scholars Program joins the initiatives of others in nurturing mentored opportunities for racial/ethnic minorities to enter the mainstream of HIV prevention research. Outreach to invite persons of minority sexual preference/orientation has further increased diversity of the trainees, making them more representative of the communities that they seek to serve with their research.

The HPTN has been deliberate, with enthusiastic NIH support, in opening up a major research consortium to young minority postdoctoral clinical, behavioral, and public health researchers to exploit HPTN data, mentors, and resourcesas well as address a historical lack of diversity within the network. The female scholar predominance may reflect the comparative dearth of minority men in research careers or the relatively large number of females in the public health field. Facilitation of the entry of minority investigators into the mainstream of NIH HIV network activity cannot engage postdoctoral trainees alone. Nurturing science and technology research skills at earlier stages of elementary and high school, college, and graduate and professional schools helps develop the pool of talent from which the research community is built. Deficits in this pipeline for underrepresented racial/ethnic minorities have been a familiar topic in the literature for many decades. ${ }^{13,31-35}$ Less well considered has been the need for persons of minority sexual orientation to also be recruited into the research community.

Scholars come from different fields, levels, and types of scientific training. One major area in which the scholars program is striving to improve is providing adequate and timely statistical support, including more rapidly delivering data to scholars. In the beginning of the HPTN scholars program, we provided support to trainees needing partial salary support, travel funds, and publication costs. More recently, we have recognized the desirability of expanding the program to include prospective scholars who do not need salary support, but need the mentoring and data access available within the HPTN. For all scholars, we seek to better nurture timely article completion, the final deliverable, but this has been delayed by the need to access analytic support and in passing the rigorous review process for network articles. The extension of the program to 18 months has permitted additional time for writing and reviewing articles. Another facilitative approach has been to recruit scholar alumni to assist new scholars who are working with the same data.

Budget logistics are also challenging, as when institutions fail to invoice expenditures within the timeline of scholar subagreements. The goal is to have monthly invoicing from scholar institutions, and the program has shown improvement in this area. We also seek to improve the engagement of mentors, given complexities of the wide geographic catchment area of the program across the United States. Colocated scholars and mentors typically interact more intensively than do those who are not located within the same city. However, this is not always feasible and creates an additional challenge. An additional mentoring innovation is that we are now recruiting HPTN scholar alumni to comentor future scholars for three reasons: (1) permit the alumni scholars to gain experience in scientific mentoring, (2) permit the current HPTN scholars to have comentorship from alumni who also are persons of color, and (3) enable the nonscholar mentor to learn from the peer mentoring dynamics. We are also considering whether mentors themselves could be trained by HPTN scholar alumni in more formal ways.

Promotion of future opportunities for scholars within the network involves their inclusion within HPTN protocol teams and scientific working groups. For example, one former scholar is a protocol team member on the HPTN 078 protocol (enhancing recruitment, linkage to care and treatment for HIV-infected MSM in the United States), whereas another is responsible for a critical intervention component within the HPTN 073 protocol (PrEP initiation and adherence among black MSM in three U.S. cities).

We recently introduced an international component to the HPTN Scholars Program (November 2015). This was based on the U.S. HPTN Scholars Program and was also reminiscent of an erstwhile, long-standing NIH Fogarty International Center Program called the AIDS International Training and Research Program. The goals are to train promising young people and also to help build research capacity at international sites. Drs. David Serwadda and Quarraisha Abdool Karim are codirectors. HPTN has enrolled six international scholars in the first three cohorts.

Within the HPTN and the NIH, there is a broad consensus that it benefits the HPTN to involve young minority HIV prevention researchers in the scientific committees, community training, writing and protocol teams, and eventually protocol chair opportunities. Enhanced minority representation in HIV research networks has improved the HPTN in many ways, increasing research productivity, representativeness of investigators, and introducing new and innovative queries and analyses. We think that many U.S. research consortia can make similar opportunities available to young investigators from underrepresented racial/ethnic and sexual minority backgrounds, breaking down historic barriers to 
access and opportunity in the research arena. Facilitating the inclusion of such researchers into the next generation of HIV scientists has the potential to improve public health by changing the quality of the questions asked and answers found as we continue to confront a U.S. HIV epidemic that is concentrated among racial/ethnic and sexual minorities.

The strong support received for this program from NIAID, complemented by topic-appropriate support from NIMH and NIDA, demonstrates the recognized importance of this effort. It will be important to collect future data on career choices, scientific foci, and productivity of the minority scientists who served as HPTN scholars to evaluate the impact of our program, as well as to better design future training and mentorship experiences to nurture careers of minority scientists within the HPTN and elsewhere. For future scholar recruitment, we will be more aggressively reaching out to southeastern and Appalachian universities, given both the magnitude of the HIV epidemic in the southern United States., as well as HIV risk from the prescription opioid and parenteral heroin epidemic. Given successes to date, we recommend the HPTN scholars model to other research networks that similarly wish to nurture the diversification of their own investigator workforces, as well as that of the HIV/AIDS field in general.

\section{Acknowledgments}

The authors wish to thank Drs. Quarraisha Abdool Karim, David Serwadda, Wafaa El-Sadr, Myron Cohen, and their NIH program officials. The HPTN is supported by the National Institute of Allergy and Infectious Diseases, National Institute on Drug Abuse, and National Institute of Mental Health (cooperative agreement nos. UM1 AI068619, UM1 AI068617, and UM1-AI068613); Centers for Innovative Research to Control AIDS, Mailman School of Public Health, Columbia University (5U1A1069466); University of North Carolina Clinical Trials Unit (AI069423); University of North Carolina Clinical Trials Research Center of the Clinical and Translational Science Award (RR 025747); University of North Carolina Center for AIDS Research (AI050410); Emory University HIV/AIDS Clinical Trials Unit (5UO1AI069418), Center for AIDS Research (P30 AI050409), and Clinical and Translational Science Award (UL1 RR025008); the Terry Beirn Community Programs for Clinical Research on AIDS Clinical Trials Unit (5 UM1 AI069503-07); and the Johns Hopkins Adult AIDS Clinical Trial Unit (AI069465) and the Johns Hopkins Clinical and Translational Science Award (UL1 RR 25005).

\section{Author Disclosure Statement}

No competing financial interests exist.

\section{References}

1. Centers for Disease Control and Prevention: HIV/AIDS: HIV by Group Atlanta: Division of HIV/AIDS Prevention, National Center for HIV/AIDS, Viral Hepatitis. Sexual transmitted diseases and tuberculosis prevention. Centers for Disease Control and Prevention. Available at www.cdc.gov/ hiv/group/index.html, accessed December 9, 2017.

2. Vermund SH, Hodder SL, Justman JE, et al.: Addressing research priorities for prevention of HIV infection in the
United States. Clin Infect Dis 2010;50(Suppl 3):S149_ S155.

3. Sista ND, Abdool Karim Q, Hinson K, et al.: Experience in international clinical research: The HIV Prevention Trials Network. Clin Investig (Lond) 2011;1:1609-1618.

4. Sullivan PS, Grey JA, Simon Rosser BR: Emerging technologies for HIV prevention for MSM: What we have learned, and ways forward. J Acquir Immune Defic Syndr 2013;63(Suppl 1):S102-S107.

5. Celum C, Baeten JM, Hughes JP, et al: Integrated strategies for combination HIV prevention: Principles and examples for men who have sex with men in the Americas and heterosexual African populations. J Acquir Immune Defic Syndr 2013;63(Suppl 2):S213-S220.

6. Mayer KH, Wheeler DP, Bekker LG, et al.: Overcoming biological, behavioral, and structural vulnerabilities: New directions in research to decrease HIV transmission in men who have sex with men. J Acquir Immune Defic Syndr 2013;63(Suppl 2):S161-S167.

7. Baeten JM, Haberer JE, Liu AY, et al.: Preexposure prophylaxis for HIV prevention: Where have we been and where are we going? J Acquir Immune Defic Syndr 2013; 63(Suppl 2):S122-S129.

8. Koblin BA, Andrasik M, Austin J: Preparing for the unexpected: The pivotal role of social and behavioral sciences in trials of biomedical HIV prevention interventions. J Acquir Immune Defic Syndr 2013;63(Suppl 2):S183-S186.

9. Rich JD, DiClemente R, Levy J, et al.: Correctional facilities as partners in reducing HIV disparities. J Acquir Immune Defic Syndr 2013;63(Suppl 1):S49-S53.

10. Shoptaw S, Montgomery B, Williams CT, et al.: Not just the needle: The state of HIV-prevention science among substance users and future directions. J Acquir Immune Defic Syndr 2013;63(Suppl 2):S174-S178.

11. Pettifor A, Bekker LG, Hosek S, et al.: Preventing HIV among young people: Research priorities for the future. J Acquir Immune Defic Syndr 2013;63(Suppl 2):S155-S160.

12. Williams C, Eisenberg $\mathrm{M}$, Becher $\mathrm{J}$, et al.: Racial disparities in HIV prevalence and risk behaviors among injection drug users and members of their risk networks. J Acquir Immune Defic Syndr 2013;63(Suppl 1):S90-S94.

13. Sopher CJ, Adamson BJ, Andrasik MP, et al.: Enhancing diversity in the public health research workforce: The research and mentorship program for future HIV vaccine scientists. Am J Public Health 2015;105:823-830.

14. Adamson BJ, Fuchs JD, Sopher CJ, et al.: A new model for catalyzing translational science: The early stage investigator mentored research scholar program in HIV vaccines. Clin Transl Sci 2015;8:166-168.

15. Nelson LE, Wilton L, Zhang N, et al.: Childhood exposure to religions with high prevalence of members who discourage homosexuality is associated with adult HIV risk behaviors and HIV infection in black men who have sex with men. Am J Mens Health 2017;11:1309-1321.

16. Hall G, Li K, Wilton $\mathrm{L}$, et al:: A comparison of referred sexual partners to their community recruited counterparts in the BROTHERS Project (HPTN 061). AIDS Behav 2015; 19:2214-2223.

17. Dyer TP, Regan R, Wilton L, et al.: Differences in substance use, psychosocial characteristics and HIV-related sexual risk behavior between Black men who have sex with men only (BMSMO) and Black men who have sex with men and women (BMSMW) in six US cities. J Urban Health 2013;90:1181-1193. 
18. Nelson LE, Wilton L, Moineddin R, et al.: Economic, legal, and social hardships associated with HIV risk among black men who have sex with men in six US cities. J Urban Health 2016;93:170-188.

19. Irvin R, Vallabhaneni S, Scott H, et al.: Examining levels of risk behaviors among black men who have sex with Men (MSM) and the association with HIV acquisition. PLoS One 2015;10:e0118281.

20. Brewer RA, Magnus M, Kuo I, et al:: Exploring the relationship between incarceration and HIV among black men who have sex with men in the United States. J Acquir Immune Defic Syndr 2014;65:218-225.

21. Brewer RA, Magnus M, Kuo I, et al:: The high prevalence of incarceration history among Black men who have sex with men in the United States: Associations and implications. Am J Public Health 2014;104:448-454.

22. Hussen SA, Stephenson R, del Rio C, et al.: HIV testing patterns among black men who have sex with men: A qualitative typology. PLoS One 2013;8:e75382.

23. Blackstock OJ, Frew P, Bota D, et al.: Perceptions of community HIV/STI risk among U.S women living in areas with high poverty and HIV prevalence rates. J Health Care Poor Underserved 2015;26:811-823.

24. Jennings L, Rompalo AM, Wang J, et al.: Prevalence and correlates of knowledge of male partner HIV testing and serostatus among African-American women living in high poverty, high HIV prevalence communities (HPTN 064). AIDS Behav 2015;19:291-301.

25. Irvin R, Wilton L, Scott H, et al.: A study of perceived racial discrimination in Black men who have sex with men (MSM) and its association with healthcare utilization and HIV testing. AIDS Behav 2014;18:1272-1278.

26. Roman Isler M, Golin C, Wang J, et al.: Venues for meeting sex partners and partner HIV risk characteristics: HIV Prevention Trials Network (HPTN064) Women's HIV Seroincidence Study (ISIS). AIDS Behav 2016;20:1208-1218.

27. Montgomery BE, Rompalo A, Hughes J, et al.: Violence against women in selected areas of the United States. Am J Public Health 2015;105:2156-2166.

28. Jennings L, Pettifor A, Hamilton E, et al:: Economic resources and HIV preventive behaviors among school-enrolled young women in rural South Africa (HPTN 068). AIDS Behav 2017;21:665-677.

29. Ritchwood TD, Hughes JP, Jennings L, et al:: Characteristics of age-discordant partnerships associated with HIV risk among young South African women (HPTN 068). J Acquir Immune Defic Syndr 2016;72:423-429.

30. Ojikutu BO, Pathak S, Srithanaviboonchai $\mathrm{K}$, et al: Community cultural norms, stigma and disclosure to sexual partners among women living with HIV in Thailand, Brazil and Zambia (HPTN 063). PLoS One 2016;11:e0153600.

31. Thakore BK, Naffziger-Hirsch ME, Richardson JL, et al.: The Academy for Future Science Faculty: Randomized controlled trial of theory-driven coaching to shape development and diversity of early-career scientists. BMC Med Educ 2014;14:160.

32. Estape-Garrastazu ES, Noboa-Ramos C, De Jesus-Ojeda L, et al:: Clinical and translational research capacity building needs in minority medical and health science Hispanic institutions. Clin Transl Sci 2014;7:406-412.

33. Ford ME, Abraham LM, Harrison AL, et al:: Mentoring strategies and outcomes of two federally funded cancer research training programs for underrepresented students in the biomedical sciences. J Cancer Educ 2016;31:228-235.

34. Reid Ponte P, Hayman LL, Berry DL, et al.: A new model for postdoctoral training: The Nursing Postdoctoral Program in Cancer and Health Disparities. Nurs Outlook 2015; 63:189-203.

35. Crockett ET. A research education program model to prepare a highly qualified workforce in biomedical and healthrelated research and increase diversity. BMC Med Educ 2014;14:202.
Address correspondence to: Sten H. Vermund Yale School of Public Health PO Box 208034

New Haven, CT 06520-8034

E-mail: sten.vermund@yale.edu 